
\title{
28 Research Suare \\ Upregulation of MMPs in Metastatic Cascade of Breast Cancer To Brain
}

\section{Mohammad Kamalabadi Farahani ( $\nabla$ kamalabadi@shmu.ac.ir )}

Shahroud University of Medical Sciences: Shahrood University of Medical Sciences https://orcid.org/0000-0002-4768-2577

\section{Amir Atashi}

Shahroud University of Medical Sciences: Shahrood University of Medical Sciences

\section{Fateme Sadat Bitaraf}

Shahroud University of Medical Sciences: Shahrood University of Medical Sciences

\section{Roqaye Karimi}

Tarbiat Modares University

Mohammad Masoud Eslami

Tarbiat Modares University

\section{Research Article}

Keywords: Triple Negative Breast Cancer, Brain Metastasis, Matrix Metalloproteinase, Zymography

Posted Date: October 19th, 2021

DOl: https://doi.org/10.21203/rs.3.rs-967695/v1

License: (c) (i) This work is licensed under a Creative Commons Attribution 4.0 International License. Read Full License 


\section{Abstract \\ Background}

Brain metastasis is a lethal complication in triple negative breast cancer (TNBC) patients. Many factors including tumor cell molecular characteristics and biological environment are the main determinant in the brain metastasis process. Matrix metalloproteinases (MMPs) play a key role in extracellular matrix degradation, implicated in numerous aspects of metastasis processes of breast cancer.

\section{Methods}

After development of syngenic animal model of TNBC, primary breast cancer cells named 4T1T were isolated from tumor mass. Highly metastatic tumor cells named 4T1B were isolated and expanded from brain metastasis lesions of cancerous mice. Quantitative real-time polymerase chain reaction and gelatinase zymography were performed to analyze the expression of MMPs in transcriptomic and proteomic level in $4 \mathrm{~T} 1 \mathrm{~T}$ and $4 \mathrm{~T} 1 \mathrm{~B}$.

\section{Results}

Our data revealed that, expression of MMPs was significantly upregulated in brain metastatic tumor cells. In transcriptomic level, MMP-2 and MMP-9 genes expression were up-regulated 4 and 3.4 folds in 4T1B, respectively. Zymographic analysis could be detect MMPs activity only in 4T1B.

\section{Conclusion}

These findings provided important insights regarding the gross alteration of MMPs expression in brain metastatic cascade of TNBC for the first time. Analysis of molecular properties of brain metastatic tumor cells can be used for understanding of molecular and genetic aspects of brain metastasis and also designing a targeted therapeutic strategies in combat with brain metastasis of TNBC.

\section{Background}

Breast cancer is the most common cancer in women worldwide[1]. Triple-negative breast cancer (TNBC) is the most aggressive and invasive type of breast cancer with poor prognosis [2]. The recurrence and metastasis of TNBC due to chemoresistance takes place in up to $70 \%$ of the patients [3]. The metastatic process of TNBC has been the subject of intense scrutiny. The brain is one of the most common organs affected in the spread of TNBC that ultimately results in fatal development of the disease.

Brain metastasis is an increasingly common complication in breast cancer patients. Approximately 15$30 \%$ of breast cancer patients develop brain metastasis [4]. The exact role of the brain environment to the 
development of the metastatic process has yet to be clarified. A suitable specific environment is important to the development of tumor cells [5]. Many theories have been developed to study and understand metastatic behavior. Factors such as neoplastic cell molecular and genetic characteristics and biological environment are thought to be determinant in the metastatic process[6]. Investigations using patient samples[7] and animal model systems of brain metastasis[8,9] are leading to improved understanding of the pathobiology of brain metastasis. Experimental models created to study the process of brain metastasis were used to isolated variants of tumor cells with enhanced brain metastatic ability. These selected variants have been used to identify and investigate the function of various genes contributing to the development of brain metastasis[10, 11].

Matrix metalloproteinases (MMPs) are a broad family of zinc-dependent proteinases that play a key role in extracellular matrix degradation, implicated in numerous pathogenic processes including cancer. Tumor cells are thought to secrete these matrix-degrading enzymes and/ or induce host cells to elaborate them[12]. In breast cancer, MMPs are thought to play an important role in invasion, metastasis and tumor angiogenesis[13]. Most reports suggest that increased expression of MMP-2 and MMP -9 proteins correlates with worse prognosis of breast cancer patients[14]. MMP2 over-expression and activation have been associated with the invasive potential of human tumors. Active MMP2 and MMP9 were detected more frequently in malignant than benign breast carcinomas [13]. MMPs have been extensively studied in the context of breast cancer prognosis. Most studies to date have been performed in human tissue collected from patients diagnosed with breast cancer or in breast cancer cell lines. In this regard, additional in vivo studies that characterize MMP expression in metastasis are needed. Few studies are available on the expression of MMPs within breast cancer metastasis [15-18]. According to our knowledge, only three studies[19-21] have characterized the expression and activity of these molecules in brain metastasis of breast cancer but alteration of MMPs in metastatic cascade of breast cancer was not focus of any of these research. It is important to determine if MMPs have different effects/roles in the development of metastasis in different organs because this may help to understand why breast cancer cells metastasize to preferential organs. Here, we focused on the metastatic process of breast cancer to the brain in a mouse model of TNBC. This model consistently produces brain metastasis to evaluate the expression and activity of MMP-2 and MMP-9 involved in metastatic cascade of breast cancer in the brain.

\section{Material And Methods}

\section{Cell culture}

4T1 cell line was obtained from the cell bank of Pasteur Institute of Iran (C604). The cells were cultured in high glucose Dulbecco's Modified Eagle's Medium (DMEM) containing 10\% FBS (fetal bovine serum) and $2 \%$ Penicillin-Streptomycin (all from Gibco, USA) in humidified atmosphere of $5 \% \mathrm{CO} 2$ at $37^{\circ} \mathrm{C}$.

\section{Induction of syngeneic animal model of breast cancer}


Female BALB/c mice weighing 20 to 25 gram obtained from Royan institute (Iran). The animals were housed in cages at 12-h photoperiod while they had free access to food and water. All animal experiments were in compliance with the relevant laws, and this study was approved by the Ethics Committee of Shahroud University of Medical Sciences (registration number: IR.SHMU.REC.1400.112). $4 T 1$ cells were subcutaneously injected to the flank (or the right hind limb) of the mice $\left(10^{5}\right.$ cells suspended in $100 \mu \mathrm{L}$ PBS) using an insulin syringe with $32 \mathrm{G}$ needle. The mice were monitored daily for the appearance and behavior characteristics.

\section{Brain metastatic and primary breast tumor cell extraction}

Primary and metastatic tumor cell extraction, was performed according to our and other group previous works[22-25]. Briefly primary tumor and brain of cancerous mice were excised after 35 days of tumor induction in mice, and surface blood was removed by rinsing it in PBS. After mincing with scissors, fragments were placed to $50 \mathrm{ml}$ conical tube. For enzymatic digestion, primary tumor and the brain were digested in $10 \mathrm{mg} / \mathrm{ml}$ collagenase type IV at $37^{\circ} \mathrm{C}$ for $75 \mathrm{~min}$ on a platform rocker. All enzymes were purchased from Sigma (St Louis, MO, USA). The digested organ filtered through 70-um cell strainers, and washed with PBS. In the next step, washed cells were resuspended in medium containing $10 \%$ FBS, 100 $\mathrm{U} / \mathrm{ml}$ Penicillin, and $100 \mathrm{ug} / \mathrm{ml}$ Streptomycin (all from Gibco, USA). Ultimately, the cells were cultured at $37^{\circ} \mathrm{C}$ in $5 \% \mathrm{CO} 2$.

\section{Quantification of MMP-2 and MMP-9 by RT-qPCR}

Primary and brain metastatic tumor cells $\left(1 \times 10^{4}\right)$ were seeded in each well of 24-well plates in complete medium. After 48 hours Total RNA was extracted from these cells using QIAzol Lysis Reagent (QIAGEN). The quality, yield, and size of extracted RNA were analyzed using spectrophotometry (NanoDropThermoFisher) and electrophoresis. The first strand cDNA synthesis was performed using reverse transcription system (Easy cDNA Synthesis Kit for RNA or mRNA to cDNA - pars tous). Real-time PCR procedure was executed based on the $1 \mathrm{ul} \mathrm{CDNA}$ in all samples. Quantization of all gene transcripts was done by SYBR Green Real time PCR Master Mix (Amplicon A/S, Denmark) using StepOnePlus ${ }^{\text {TM }}$ Real-Time PCR System, according to the manufacturer's instruction. The amplification procedure was as follows: 1 cycle of $95^{\circ} \mathrm{C}$ for $15 \mathrm{~min}, 40$ cycles of $95^{\circ} \mathrm{C}$ for $30 \mathrm{sec}, 60^{\circ} \mathrm{C}$ for $30 \mathrm{sec}$, and $72^{\circ} \mathrm{C}$ for $30 \mathrm{sec}$. The exact mRNA expression was normalized to the expression level of GAPDH. Relative changes of gene expression were calculated by the following formula, and the data was represented as fold up-regulation/downregulation.

Fold change $=2^{-\Delta \Delta \mathrm{Ct}}$, where $\Delta \Delta \mathrm{Ct}=$ [Ct of MMPS (in treated cells) $-\mathrm{Ct}$ of GAPDH (in treated cells) - [Ct of MMPS (in control cells) - Ct of GAPDH (in control cells)].

Primers were designed using AllelelD version 6 software (Premier Biosoft Inc.).

The used primers are as follows: 
For MMP-2, Forward 5'-TTTATTTGGCGGACAGTGAC-3', Reverse 5'- AGTTAAAGGCAGCATCTACTTG -3';

For MMP-9, Forward 5'-TCCAGTATCTGTATGGTCGTG-3', Reverse 5'- CATAGTGGGAGGTGCTGTC -3';

For GADPH, Forward 5'-CCTGGAGAAACCTGCCAAGTA-3', Reverse 5'-GGCATCGAAGGTGGAAGAGT -3'.

\section{Zymography}

Zymography was performed on $9 \%$ polyacrylamide gels that had been cast in the presence of gelatin. Briefly, samples $(100 \mu \mathrm{l})$ were resuspended in loading buffer and separated on a 9\% SDS-PAGE gel containing $0.5 \mathrm{mg} / \mathrm{ml}$ gelatin without prior denaturation. After electrophoresis, the gels were washed to remove SDS and incubated for $30 \mathrm{~min}$ at room temperature in a renaturing buffer ( $50 \mathrm{mM}$ Tris, $5 \mathrm{mM}$ $\mathrm{CaCl} 2$, and $1 \%$ Triton $\mathrm{X}-100)$. The gels were incubated for $48 \mathrm{~h}$ at $37^{\circ} \mathrm{C}$ in a developing buffer (50 mM Tris$\mathrm{HCl}[\mathrm{pH} 7.8], 5 \mathrm{mM} \mathrm{CaCl} 2,0.15 \mathrm{M} \mathrm{NaCl}$, and $1 \%$ Triton X-100) and then stained with Coomassie Brilliant Blue G-250, destained in $30 \%$ methanol, and flooded with $10 \%$ acetic acid to detect gelatinase secretion.

\section{Statistical analysis}

Results are expressed as the mean \pm standard deviation. Data were analyzed with GraphPad Prism statistical software 6.0 (GraphPad Software, La Jolla, CA, USA) using Paired Samples t Test. P $<0.05$ was considered statistically significant.

\section{Results}

\section{Primary and metastatic tumor cells extraction}

Metastatic animal model of breast cancer was generated after 35 days following tumor induction in Balb/c mice (Figure 1A). When injected into BALB/c mice, 4T1 spontaneously produces highly metastatic tumors that can metastasize to the brain while the primary tumor is growing in situ. The primary tumor does not have to be removed to induce metastatic growth. $\mathrm{H}$ and $\mathrm{E}$ staining and pathological confirmation were performed on tumor tissues and brain metastatic lesions (Figure 1C, B). We properly extracted primary and brain metastatic tumor cells from subcutaneous primary tumor and brain of cancerous mice, respectively (Figure 1C, B). The metastatic tumor cells in the brain, after primary isolation, form colonies in the culture medium. Due to the high rate of growth and proliferation, the tumor cells in these colonies are purified after 3 passages. These tumor cells are called brain metastatic tumor cells or 4T1B while tumor cells that are obtained in the same way, from the original tissue of the tumor, are primary tumor cells called $4 \mathrm{~T} 1 \mathrm{~T}$ (Figure $1 \mathrm{C}, \mathrm{B}$ ).

\section{Significant Upregulation of MMPs in brain metastatic tumor cells in mRNA level}

The expression of matrix metalloproteinase (MMP-2 and MMP-9), was analyzed in 4T1T and 4T1P. The quality, yield, and size of extracted RNA, synthesized cDNA, and PCR products were confirmed using nanodrope and gel electrophoresis. As shown in fig 2 the expression of MMP-9 was up-regulated 3.4 
times in 4T1P compared with 4T1T. About MMP-2, the expression of these MMP was involved in higher alteration in metastatic cascade of breast cancer and up-regulated 4 times in 4T1P compared with 4T1T (Figure 2).

\section{Zymographic analysis could be detect MMPs protein expression only in 4T1B}

Gelatin zymography was used to evaluate gelatinase activity, especially MMP-9 and MMP-2. MMPs activity were observed based on the white band formed on the SDS-page gel, which indicated the degradation of gelatin by MMPs. The result shows that detection of gelatinase activity of MMPs was feasible only in 4T1B (Figure 3). As showed in fig 3 results indicated that secretion of MMP proteins in conditioning media (CM) of $4 \mathrm{~T} 1 \mathrm{~T}$ was not as enough as to detect in zymography but in $4 \mathrm{~T} 1 \mathrm{~B}$, secreted MMPs in CM could be create a white band on SDS-page.

\section{Discussion}

In the present study, we revealed significant increase in MMP-2 and MMP-9 expression in brain metastatic tumor cells in mRNA and protein levels. Our works report the gross alteration of MMPs expression in brain metastatic cascade of TNBC for the first time and suggesting that these molecules may be relevant in the metastatic process of breast cancer to the brain. To our knowledge, this is the first report of characterization of these molecules in brain metastasis cascade of breast cancer.

Many reports correlating MMP activity with metastatic and invasive behavior of tumor cells [12]. Previous studies that describe MMP expression correlated with breast cancer metastasis reveal that MMPs may be important for the metastatic process[16]. About breast cancer brain metastasis, incidence of metastasis to the brain was increased in animals injected intracardiac with clones of breast cancer cells transfected with MMP2[18]. Results of research by Mendes et al in 2005 showed that MMP-2, -3 and -9 proteins expressions are significantly higher in neoplastic brain tissue compared to normal brain tissue[21].

The determination of what cell component of the tumor mass expresses MMPs is important in order to understand the role of these molecules in tumor development. Some studies have localized MMP2 to neoplastic epithelial cells. Others, however, associate them with different components of the tumor stroma[26] and/or angiogenic blood vessels[27]. MMP9 has been associated with neoplastic cell plasma

membrane[28], non-neoplastic ducts and acini, stromal fibroblasts; endothelial cells, and tumor-infiltrating inflammatory cells including neutrophils, macrophages, and lymphocytes. Expression of MMP3 was observed in both tumor and stroma cells[29]. In concurrence with previous reports we observed high level of MMP2 and MMP9 expression in metastatic tumor cells.

MMP inhibitors are being investigated as an important tool for cancer treatment [30, 31]. In a study for determination of MMPs role in breast cancer brain metastasis development, application of a selective MMPs inhibitor induce slight but significant decrease in in vitro ENU 1564 invasion behavior when cells were in presence of MMPs inhibitor. Additionally development of brain metastasis in animals treated with MMPs inhibitor was decreased dramatically. According to in vivo results, this group strongly suggests 
that MMPs are important in the brain metastatic process of breast cancer. But for interpretation of disparity observed in vivo vs in vitro results this group emphasized low levels of in vitro MMP expression [21]. In opposition with these results, our work in, in vitro level detect a high level of MMPs expression in metastatic breast tumor cells.

Liu etal in in a xenograft model revealed the role of MMP-1 in breast cancer growth and metastasis to the brain. In this study two variants of the MDA-MB-231 human breast cancer cell line selected for enhanced ability to form brain metastases in nude mice (231-BR and 231-BR3 cells) were found to express high levels of matrix metalloproteinase-1 (MMP-1) [19]. In a recent study, after isolation of brain metastatic tumor cells from brain of cancerous mice, results indicated that silencing mir-202-3p increases MMP-1 and promotes a brain invasive phenotype in these cells [20].

In conclusion our study, for the first time, use a mouse model for distant breast cancer metastasis to the brain to successfully study expression and activity of MMP-2 and MMP -9. The use of animal models to study in vivo tumor progression and metastatic behavior is important to understand the mechanism of metastasis development. It is also an important tool for pharmacological evaluation of cancer therapy. Several synthetic MMP inhibitors are under investigation for clinical trials in patients with cancer. They are thought to inhibit both primary tumor invasion and metastasis[30, 31]. Our results indicate that MMPs may be involved in breast cancer metastasis to the brain. By targeting these molecule we can overcome these resistant and preventing recurrence of the disease in patients with metastatic breast cancer. This understanding may be utilized in the development of the current therapeutic approach to metastatic cancer.

\section{Declarations}

\section{Acknowledgments:}

We want to especially thank Dr.vasei and Dr.ahmadbeigi for his practical advisation and helpful information about breast cancer.

\section{Compliance with Ethical Standards:}

\section{Conflict Of Interest}

The author declares that they have no competing interests.

\section{Ethical statement}

This study was approved by the Ethics Committee of Shahroud University of Medical Sciences (registration number: IR.SHMU.REC.1400.112).

\section{Funding}


This work was supported by a grant from the Shahroud University of Medical Sciences (SHMU) Grant No 963.

\section{Author contributions:}

Conceptualization, M-KF; methodology, M-KF, AA, FS-B, RK and MM-E; formal analysis M-KF, RK and MME; writing-original draft preparation, MKF; writing-review and editing, AA; supervision, M-KF.

\section{Data availability:}

All data generated or analysed during this study are included in this published article.

\section{References}

1. Siegel RL, Miller KD, Jemal A (2016) Cancer statistics, 2016. Cancer J Clin 66:7-30

2. Yao H, He G, Yan S, Chen C, Song L, Rosol TJ, Deng X (2017) Triple-negative breast cancer: is there a treatment on the horizon? Oncotarget 8: 1913

3. Isakoff SJ (2010) Triple negative breast cancer: role of specific chemotherapy agents. Cancer journal (Sudbury Mass) 16:53

4. Rusciano D, Burger MM (1992) Why do cancer cells metastasize into particular organs? Bioessays 14:185-194

5. Hall DG, Stoica G (1994) Characterization of brain and bone-metastasizing clones selected from an ethylnitrosourea-induced rat mammary carcinoma. Clin Exp Metastasis 12:283-295

6. Nishizuka I, Ishikawa T, Hamaguchi Y, Kamiyama M, Ichikawa Y, Kadota K, Miki R, Tomaru Y, Mizuno $\mathrm{Y}$, Tominaga N (2002) Analysis of gene expression involved in brain metastasis from breast cancer using cDNA microarray. Breast Cancer 9:26-32

7. Palmieri D, Fitzgerald D, Shreeve SM, Hua E, Bronder JL, Weil RJ, Davis S, Stark AM, Merino MJ, Kurek R (2009) Analyses of resected human brain metastases of breast cancer reveal the association between up-regulation of hexokinase 2 and poor prognosis. Mol Cancer Res 7:1438-1445

8. Bos PD, Zhang XH-F, Nadal C, Shu W, Gomis RR, Nguyen DX, Minn AJ, van de Vijver MJ, Gerald WL, Foekens JA (2009) Genes that mediate breast cancer metastasis to the brain. Nature 459:10051009

9. Fidler IJ, Balasubramanian K, Lin Q, Kim SW, Kim S-J (2010) The brain microenvironment and cancer metastasis. Mol Cells 30:93-98

10. Kim LS, Huang S, Lu W, Lev DC, Price JE (2004) Vascular endothelial growth factor expression promotes the growth of breast cancer brain metastases in nude mice. Clin Exp Metastasis 21:107118

11. Yoneda T, Williams PJ, Hiraga T, Niewolna M, Nishimura R (2001) A bone-seeking clone exhibits different biological properties from the MDA-MB-231 parental human breast cancer cells and a brain-seeking clone in vivo and in vitro. Journal of bone mineral research 16:1486-1495 
12. Stetler-Stevenson WG, Aznavoorian S, Liotta LA (1993) Tumor cell interactions with the extracellular matrix during invasion and metastasis. Annu Rev Cell Biol 9:541-573

13. Hynes RO (2003) Metastatic potential: generic predisposition of the primary tumor or rare, metastatic variants-or. both? Cell 113:821-823

14. La Rocca G, Pucci-Minafra I, Marrazzo A, Taormina P, Minafra S (2004) Zymographic detection and clinical correlations of MMP-2 and MMP-9 in breast cancer sera. British journal of cancer 90:14141421

15. Yoneda T (2000) Cellular and molecular basis of preferential metastasis of breast cancer to bone. Journal of Orthopaedic Science 5:75-81

16. Ohshiba T, Miyaura C, Inada M, Ito A (2003) Role of RANKL-induced osteoclast formation and MMPdependent matrix degradation in bone destruction by breast cancer metastasis. British journal of cancer 88:1318-1326

17. Scott K, Holdsworth H, Balkwill F, Dias S (2000) Exploiting changes in the tumour microenvironment with sequential cytokine and matrix metalloprotease inhibitor treatment in a murine breast cancer model. British journal of cancer 83:1538-1543

18. Tester AM, Waltham M, Oh S-J, Bae S-N, Bills MM, Walker EC, Kern FG, Stetler-Stevenson WG, Lippman ME, Thompson EW (2004) Pro-matrix metalloproteinase-2 transfection increases orthotopic primary growth and experimental metastasis of MDA-MB-231 human breast cancer cells in nude mice. Cancer research 64:652-658

19. Liu H, Kato Y, Erzinger SA, Kiriakova GM, Qian Y, Palmieri D, Steeg PS, Price JE (2012) The role of MMP-1 in breast cancer growth and metastasis to the brain in a xenograft model. BMC Cancer 12:111

20. Harati R, Hafezi S, Mabondzo A, Tlili A (2020) Silencing miR-202-3p increases MMP-1 and promotes a brain invasive phenotype in metastatic breast cancer cells. PloS one 15:e0239292

21. Mendes O, Kim H-T, Stoica G (2005) Expression of MMP2, MMP9 and MMP3 in breast cancer brain metastasis in a rat model. Clin Exp Metastasis 22:237-246

22. Walsh C, Tanjoni I, Uryu S, Tomar A, Nam J-O, Luo H, Phillips A, Patel N, Kwok C, McMahon G (2010) Oral delivery of PND-1186 FAK inhibitor decreases tumor growth and spontaneous breast to lung metastasis in pre-clinical models. Cancer Biol Ther 9:778-790

23. FARAHANI MK (2020) High Capacity of the Metastatic Breast Tumor Cells in Sphere Formation: Clue for Chemoresistance in Triple-Negative Breast Cancer. TURKISH JOURNAL OF ONCOLOGY 1

24. Kamalabadi-Farahani M (2020) Down-Regulation of Death Receptor-5 in Metastatic Cascade of Triple-Negative Breast Cancer. TURKISH JOURNAL OF ONCOLOGY 1

25. Kamalabadi-Farahani M, Najafabadi H, Jabbarpour MR Z (2019) Apoptotic Resistance of Metastatic Tumor Cells in Triple Negative Breast Cancer: Roles of Death Receptor-5. Asian Pac J Cancer Prev 20:1743-1748. doi:10.31557/apjcp.2019.20.6.1743

26. Caudroy S, Polette M, Tournier J-M, Burlet H, Toole B, Zucker S, Birembaut P (1999) Expression of the extracellular matrix metalloproteinase inducer (EMMPRIN) and the matrix metalloproteinase-2 in 
bronchopulmonary and breast lesions. Journal of Histochemistry Cytochemistry 47:1575-1580

27. Bartsch JE, Staren ED, Appert HE (2003) Matrix metalloproteinase expression in breast cancer. J Surg Res 110:383-392

28. Balduyck M, Zerimech F, Gouyer V, Lemaire R, Hemon B, Grard G, Thiebaut C, Lemaire V, Dacquembronne E, Duhem T (2000) Specific expression of matrix metalloproteinases 1, 3, 9 and 13 associated with invasiveness of breast cancer cells in vitro. Clin Exp Metastasis 18:171-178

29. Brummer $O$, Athar $S$, Riethdorf L, Löning T, Herbst $H$ (1999) Matrix-metalloproteinases 1,2 and 3 and their tissue inhibitors 1 and 2 in benign and malignant breast lesions: an in situ hybridization study. Virchows Arch 435:566-573

30. Nozaki S, Sissons S, Chien D-S, Sledge GW (2003) Activity of biphenyl matrix metalloproteinase inhibitor BAY 12-9566 in a human breast cancer orthotopic model. Clin Exp Metastasis 20:407-412

31. Lee S, Sakurai H, Oshima K, Kim S, Saiki I (2003) Anti-metastatic and anti-angiogenic activities of a new matrix metalloproteinase inhibitor, TN-6b. Eur J Cancer 39:1632-1641

\section{Figures}

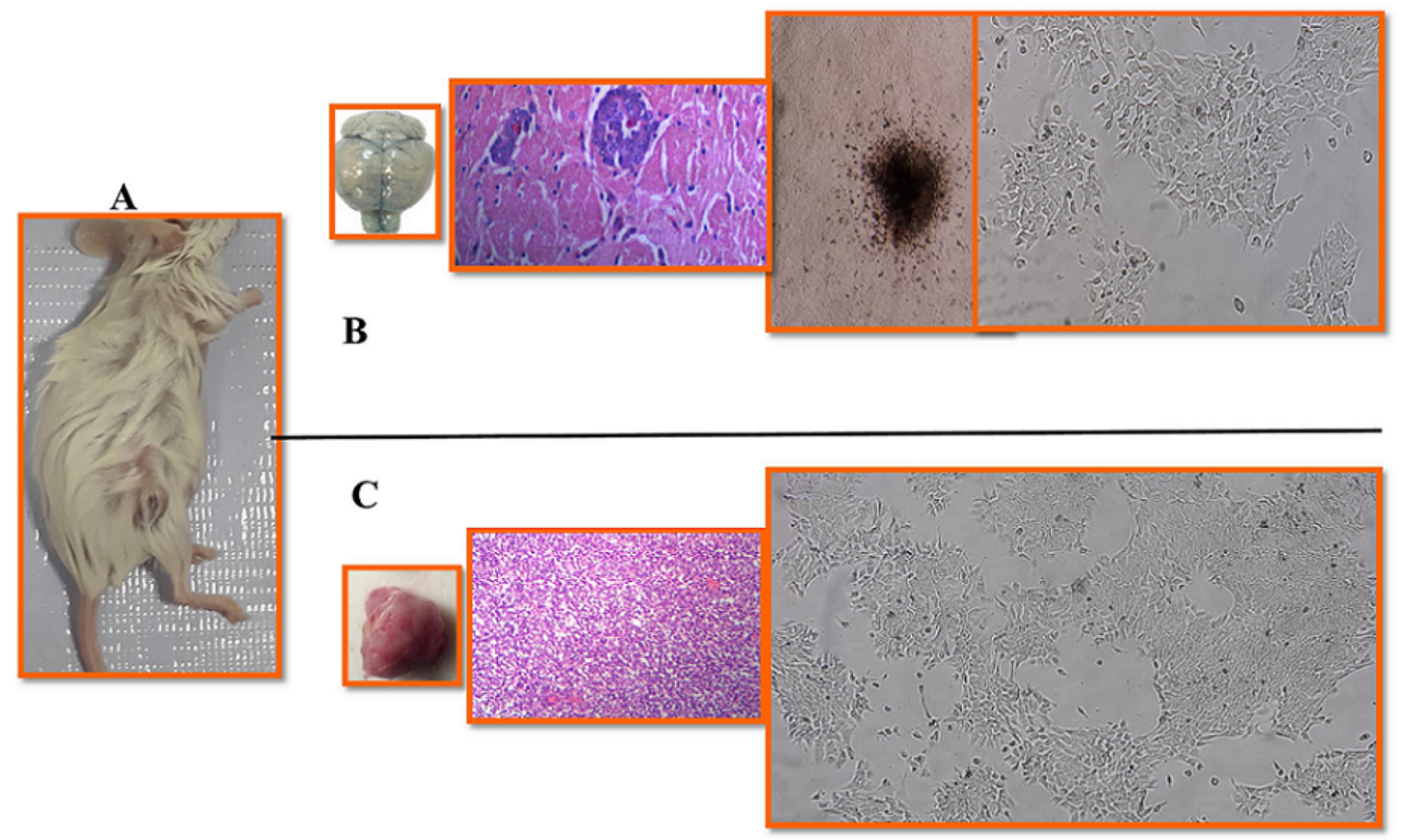

\section{Figure 1}

Primary and Brain Metastatic Tumor Cells Isolation. A. Metastatic animal model of triple negative breast cancer was generated after 35 days of tumor induction in Balb/c mice. B. Brain metastatic tumor 
isolation, $\mathrm{H} \& \mathrm{E}$ staining and metastatic tumor cell extraction was performed on brain of cancerous mice. C. Primary tumor isolation, H\&E staining and primary tumor cell extraction was performed on primary tumor tissues.

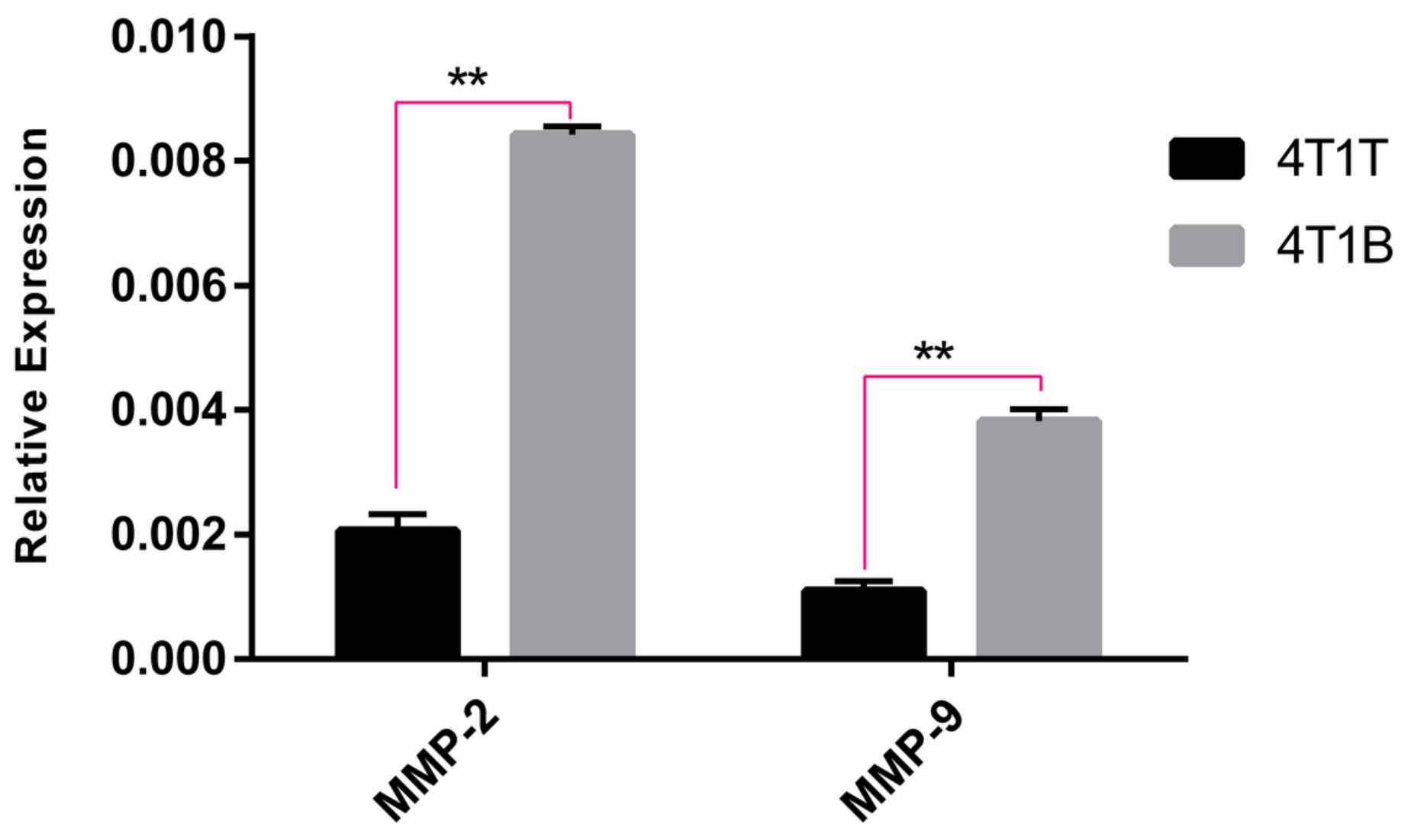

Figure 2

Enhanced Expression of MMPs in Brain Metastatic Tumor Cells Using Real-Time PCR. Both MMP-2 and MMP-9 was significantly upregulated in Brain Metastatic Tumor Cells. All results are expressed as mean \pm SD from at least three independent experiments analyzed by Two-tailed $T$ test. $* \star P<0.001$. 


\section{$4 T 1 T) 4 T 1 B$}

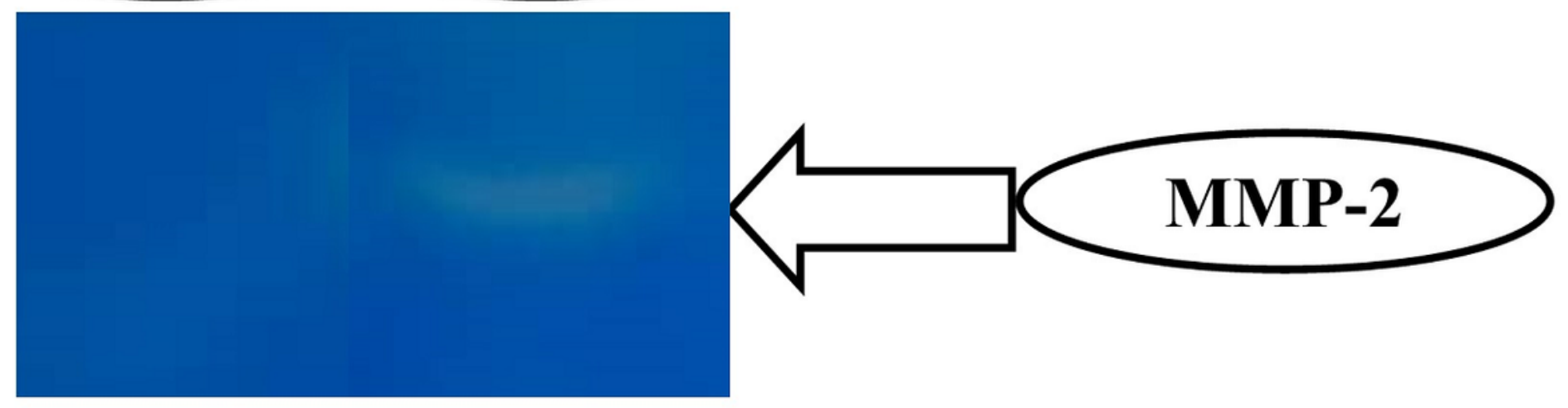

Figure 3

Gelatinolytic Activity of MMPs in Brain Metastatic Tumor Cells. Detection of gelatinase activity of MMPs was feasible only in 4T1B. 